\title{
Feature Identification of Hepatic Cancer Ultrasound Image using Gaussian Filtering Combined with Intensity Adjustment
}

\author{
${ }^{1)}$ Budi Utami Fahnun, ${ }^{2)}$ A. Benny Mutiara, ${ }^{3)}$ Johan Harlan, ${ }^{4)}$ Eri Prasetio Wibowo \\ Faculty of Computer Science and Information Technology, \\ Gunadarma University, Depok, Indonesia
}

\begin{abstract}
Ultrasound images are widely used in medical domain due to its relatively low cost, non-invasive characteristics, and real-time imaging capabilities. Ultrasound images have grayscale color values that are susceptible to development of speckle noise in the image. Medical diagnostic images are usually corrupted by noise during their acquisition and most of the noise is speckle noise. Improved preserved features are very important in medical ultrasound images of liver cancer and we need an image enhancement process to achieve the aims. In this research we improve the efficiency of denoising and maintain shape features and texture features properly by using Gaussian Filtering and Adjustment Intensity methods. The proposed approach also improve visual quality of the ultrasound images. The enhancement techniques is to bring out detail that is obscured, or simply to highlight certain features of interest in an image The results demonstrate the ability of proposed method for noise suppression as well as feature and edge preservation in terms of a variety of performance measures.
\end{abstract}

Keywords-Gaussian Filter; Histogram Equalization; Image Enhancemen; Intensity Adjustment; Ultrasound image

\section{INTRODUCTION}

Medical image processing must have high accuracy because an image processing is very accommodating for the medical treatment process at the clinic or hospital. In the medical image it is necessary to be able to see the smallest and most disguised structure of the human body. Misunderstanding data is very fatal, because it can threaten patient safety so that it can be considered as a doctor's practice that violates the code of ethics. Therefore, it is necessary to have an accurate visual understanding of data so that experts can identify abnormalities such as liver organ with high accuracy. Ultrasonograph is a device used to capture images by using pulses ultrasound waves emitted by the transducer with certain frequency and reflected back on the sensor. Ultrasound imaging is mostly preferred because of its non-invasive characteristics, low cost and real-time imaging capabilities, and portability. But since the texture of normal and abnormal regions is intermixed, correct interpretation of ultrasound images are difficult and inexperience examiner may end in interpreting them incorrectly, as the analysis of these images is very complex [1]. The complex nature of ultrasound images may cause the diagnosis be prone to errors and heavily based upon the doctors and radiologists experience and expertise. Therefore, detecting and enhancing the boundaries between cavities and organs is greatly needed in ultrasound imaging. Ultrasound images also contains echo disorders called speckles. Speckle is multiplicative noise with undesired interference effects on images. To improve feature quality of the images, the most important problem is the reduction of the speckles. The speckle reduction process aims to enhance important details and features of ultrasound images, so the speckle reduction algorithm must be designed in a certain way without losing meaningful information such as texture features and edge features[2]. The idea behind enhancement techniques is to bring out detail that is obscured, or simply to highlight certain features of interest in an image. Image enhancement alters an image to makes its meaning clearer to human observers. Image enhancement is the process by which we try to improve an image so that it looks subjectively better.

Research conducted [3-4] mentions that image enhancement is a method of improving the definition of a video picture by a computer program, which reduces the lowest grey values to black and the highest to white used for pictures from microscopes, surveillance cameras and scanners.

According Ogrenci [6], image enhancement is often used to improve the image quantity by emphasizing some information and weakening some unnecessary information of an image according to 'users' need. The results of research conducted by Mittal [7], are show the image enhancement processes consist of a collection of techniques that seek to improve the visual appearance of an image and to convert the image into a better form suited for analysis by a human or a machine.

In this research, the operating method will be used for point intensity adjustment and histogram equalization technique. Intensity adjustment works by doing a linear mapping of intensity values on the initial histogram to intensity values on the new histogram. The histogram equalization technique aims to produce an output image that has a relatively similar histogram value. The results obtained sometimes have a low light intensity and produce various noise in the image. From these results, it takes an image processing process to improve the quality image so that results can be seen more clearly and accurately by using certain methods.

This study aims to improve ultrasound images of hepatic cancer so the details and important features can be seen clearly by human eyes. 
The image processing includes segmentation, feature extraction, and classification.

\section{PREPROCESSING METHODS}

\section{A. Preprocessing}

Objects and structures in the body have various physical contrasts, these conditions will be reflected in visualization of contrast seen from medical images. Physical contrast is different at every place in the body's tissues or organs. Physical characteristics to produce visualization of contrast appear from medical images will be different in each image modality used. Image processing is a processing process where input and output are in the form of images or a set of characteristics or parameters related to the image. Digital image processing is generally defined as two-dimensional image processing with a computer and includes all twodimensional data. One part of image processing is preprocessing. Preprocessing is done to reduce noise, contrast settings, and image sharpness settings. The enhancement includes denoising and contrast techniques. The contrast enhances techniques performed through some operations like Point operations are referred to as gray-level transformations or spatial transformations.

\section{B. Gaussian Filtering}

Gaussian filtering is used to blur the image, eliminating noise and detail. Gaussian filtering is more effective in smoothing the image based on the human visual perception system [3]. Dimension the gaussian formula is:

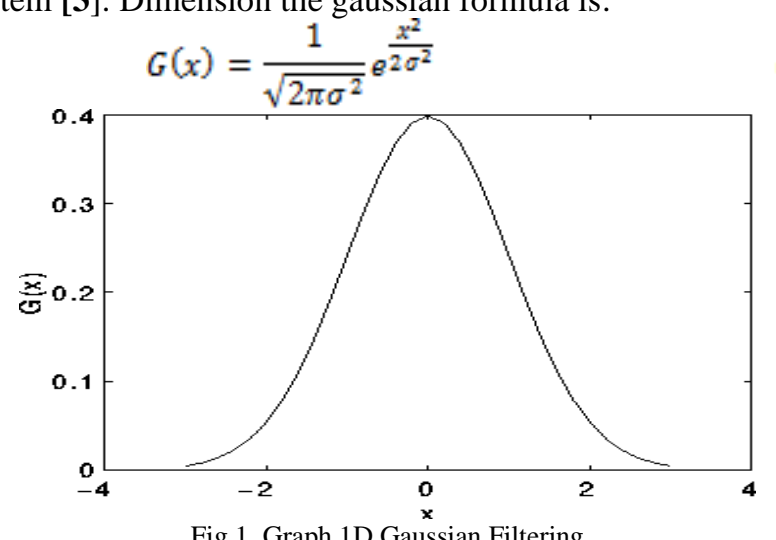

Where $\sigma$ is the standard deviation of the distribution. Distribution is assumed to have an average of 0 . Graphically the display is 1-dimensional based on the $\mathrm{X}$-axis in fig. 1 . The time related to the image is needed to use a two-dimensional Gaussian Function which is a product of two Gaussian 1D functions that are combined between $\mathrm{x}$ and $\mathrm{y}$ [3]. The formula is:

$$
G(x, y)=\frac{1}{\sqrt{2 \pi \sigma^{2}}} e^{\frac{x^{2}+y^{2}}{2 \sigma^{2}}}
$$

On the representation of $2 \mathrm{D}$ graph in gaussian with an average axis value $(0.0)$ and a value for $\sigma=1$ shown to the fig. 2 .

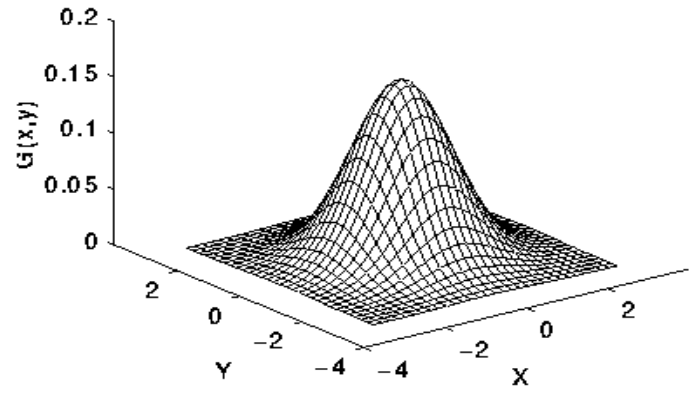

Fig 2. Graph 2D Gaussian Filtering

\section{Intensity Adjustment}

Intensity Adjusment is a technique for mapping the intensity value of an image. Intensity adjusment works by doing linear mapping of the intensity value on the initial histogram to an intensity value on the new histogram. Brightness is a visual perception of reflected light, increased brightness refers to an increase in image illumination [4]. The formula of brightness and contrast is:

$$
F=\frac{259(C+255)}{255(259-C)}
$$

In order for the algorithm to function correctly, the value for the contrast correction factor $(\mathrm{F})$ needs to be stored as a float number and not as an integer. Then the value of $\mathrm{C}$ in the formula shows the desired level of contrast. The brightness value will usually be in the range of -255 to +255 for a 24-bit palette. Negative values darken the image and, conversely, positive values will brighten the image. In Figure 3 is an example of improving image ultrasoud liver cancer with Intensity Adjustment.
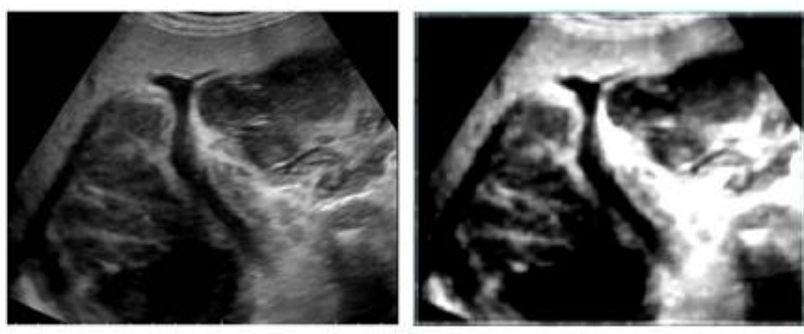

Fig 3. Example results process from Intensity Adjustment

On Intensity Adjusment can also do gamma correction by mapping low down, and high up. By default, the value between low and high is mapped linearly to the value between bottom and top. Gamma is represented by Greek letters, can be described as the relationship between the input and output produced [3]. For the scope of the input value will be the intensity value of an image. In this case the relationship between input / output is output value proportional to the input raised to gamma power. The formula for calculating the results produced is as follows;

$$
I^{y}=255 \times\left(\frac{I}{255^{2}}\right)^{y}
$$




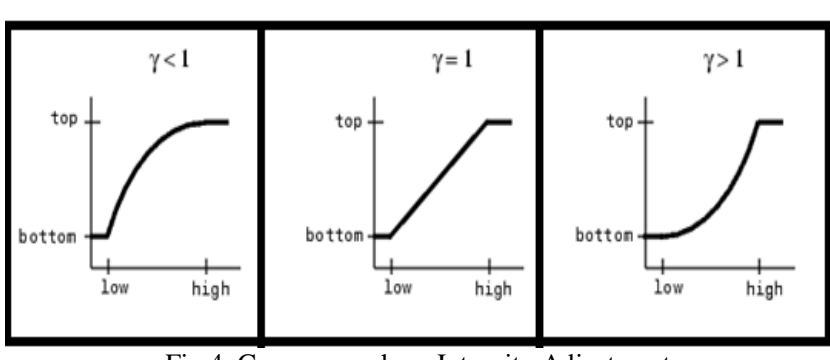

Fig 4. Gamma graph on Intensity Adjustment

The range of values used for gamma will depend on the application, but what is usually used is the value using the range 0.01 to 7.99 .

\section{Histogram Equalization}

- Images with poor contrast usually contain unevenly distributed gray values

- Histogram Equalization is a method for stretching the contrast by uniformly distributing the gray values

enhances the quality of an image

useful when the image is intended for viewing

- not always useful for image processing

Histogram equalization is a method for processing images to adjust image contrast by modifying the histogram intensity distribution. The aim of this technique is to provide linear trends to the cumulative probability functions associated with the image [2]. Histogram equalization processing depends on the use of a cumulative probability function (cdf). Cdf is the cumulative number of all the probabilities that exist in the domain and is determined by:

$$
\operatorname{cd} f(x)=\sum_{k=-\infty}^{x} P(k)
$$

The idea of this process is to give to the image the resulting cumulative linear distribution function. Fig. 5. Graphic value of Histogram Equalization. Linear Cdf is associated with a value histogram equal to the resulting image.
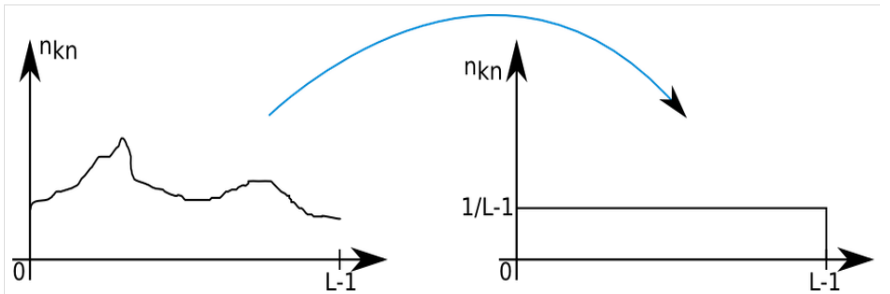

Fig. 5. Graphic value of Histogram Equalization

A histogram is a distribution with a discrete intensity level in the range [0, L-1]. Distribution is a discrete function $\mathrm{h}$ that corresponds to each intensity level: rk with the number of intensity pixels: nk. Normalizing histograms is a technique that consists of transforming a discrete distribution of intensity into a discrete probability distribution. To do this, it is necessary to divide each histogram value by the number of pixels. Because a digital image is a set of discrete values that can be seen as a matrix and it's the same as dividing nk each by the dimensions of the array which is the product of the width by the length of the image. The formula is:

$$
n_{k n}=\frac{n k}{\text { length } \times \text { width }}=\operatorname{Pr}\left(n_{k}\right)
$$

\section{RESULT AND ANALYSIS}

This study uses data from the ultrasound liver cancer website [5-8]. The image used as the study material is an abnormal liver image, HCC image, ICC image and metastases image. The purpose of this study is to increase the intensity of the image so that the image feature details will be clearly visible. Thus the resulting image can be used at a later stage. The flow of the research process is shown in fig. 6 which includes the image acquisition stage and the preprocessing stage. Preprocessing using the Gaussian filtering method and Intensity Adjustment and displaying a histogram of each experiment. In testing to determine the intensity value of image improvement with preprocessing using the Gaussian filtering method and Intensity Adjustment based on Matlab 2017a programming and displaying a histogram of each experiment.

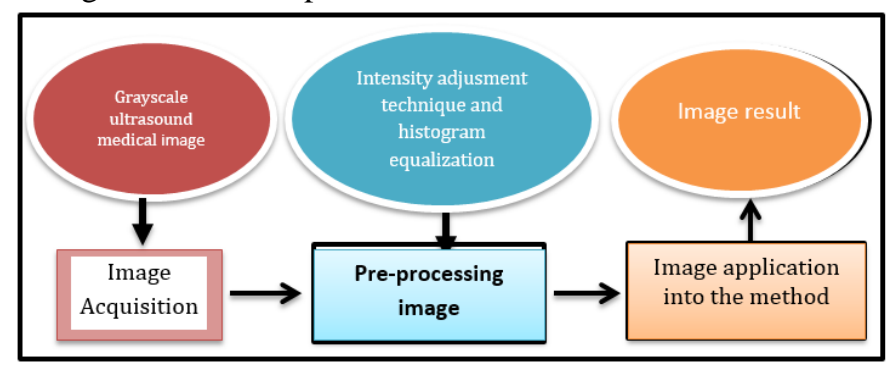

Fig. 6. Proposed research chart

\section{A. Implementation}

Creating a GUI image preprocessing program using matlab programming that will be used to increase the intensity of an image and get the value of the change. GUI images preprocessing process can be seen on fig. 7 below;

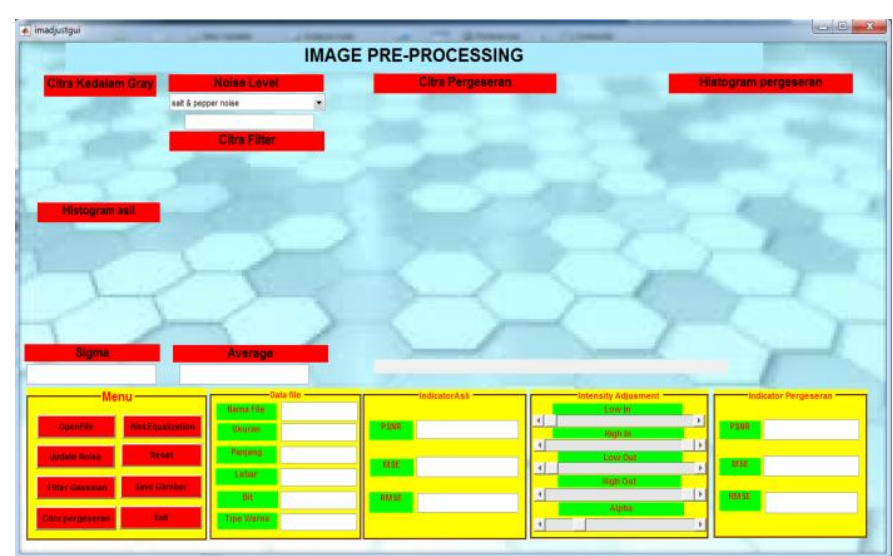

Figure 7. GUI Image Preprocessing program

In the program there are 2 indicator values namely the value of the initial input image and the shift image as the output image which will later be seen in comparison with the calculation requirements whether the original image value is much different from the output value. Result of this process can see in fig 8 below; 


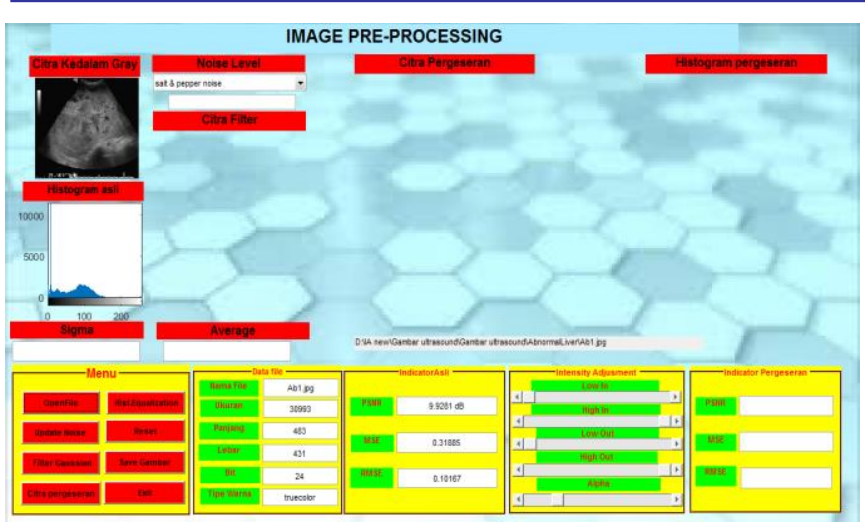

Figure 8. Process of initial image input, initial histogram and initial value.

The first step is to open the image to be processed, then the indicator value will appear from the original image which will be compared to the output image. Providing noise and filtering processes is seen in the fig. 9 below;

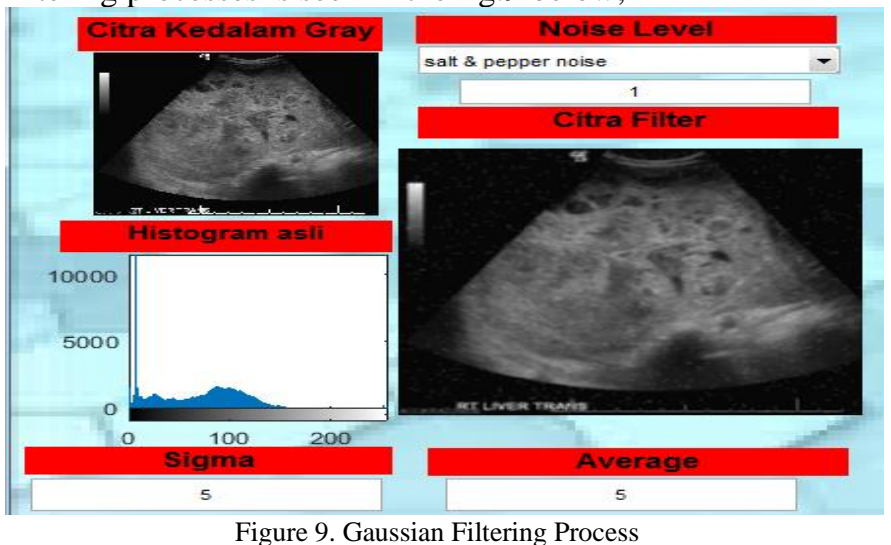

After that, noise updates were performed using salt \& pepper noise with a level 1 value which was then filtered by image based on the value of sigma and the average of 5, so that the image looked smoother as shown in fig. 10 below;

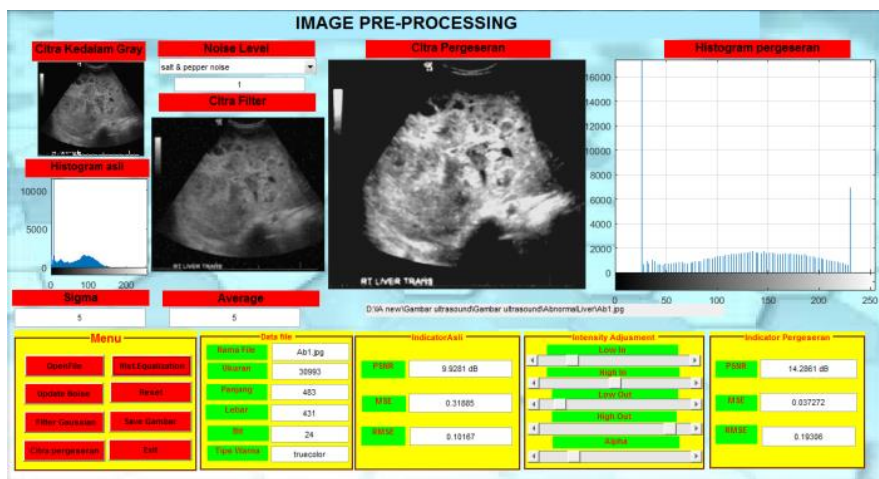

Figure 10. Overall results and increased intensity

The final stage increases the intensity of light based on the filtered image and sees changes in the difference in histogram and the value of the initial and final indicators.

\section{B. Calculation of MSE, RMSE and PSNR Value}

Process results are obtained after the testing process and produce values from MSE, RMSE and PSNR. Mean Square Error (MSE), Root Mean Squared Error (RMSE), and Peak
Signal-to-Noise Ratio (PSNR) are examples of parameters commonly used as indicators to measure the similarity of two images. These parameters are often used to compare the results of image processing with the original image or original image [8]. The equation used to calculate the three parameters is as follows:

$$
\text { MSE }=\frac{1}{m \times n} \sum_{i=0}^{n-1} \sum_{j=0}^{m-1}\left[f\left(i_{i} j\right)-g\left(i_{i} j\right)\right]^{2}
$$

RMSE $=\sqrt{\frac{1}{m \times n} \sum_{i=0}^{n-1} \sum_{j=0}^{m-1}\left[f\left(i_{i} j\right)-g\left(i_{i} j\right)\right]^{2}}$

$$
P S N R=10 \log _{10} \frac{255^{2}}{M S E}
$$

MSE and RMSE have no units while the units of PSNR are decibels. The more similar the two images, the MSE and RMSE values are getting closer to zero. Whereas in the PSNR, two images are said to have a low level of similarity if the PSNR value is above $30 \mathrm{~dB}$. The following are examples of each of the results of the comparison of the values of 2 images, namely the initial image and the final image. From the 5 samples above, after doing image enhancement, the two images have a value that is still relatively the same which indicates that it does not change significantly to the initial image. The samples are of 5 types namely Abnormal, HCC, ICC, Metastatic and Normal. In the table. 1 is the result of quality testing with the Calculation of MSE, RMSE and PSNR Value.

Table. 1. the result of quality testing with the Calculation of MSE, RMSE and PSNR Value

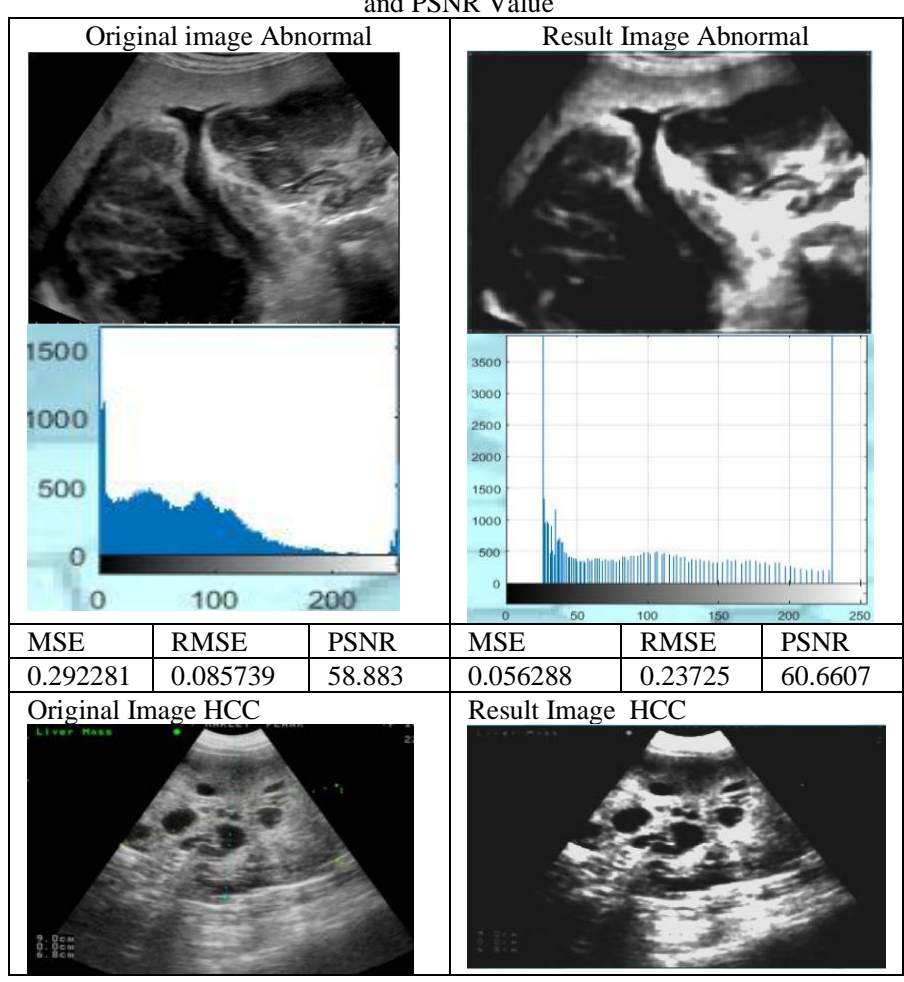




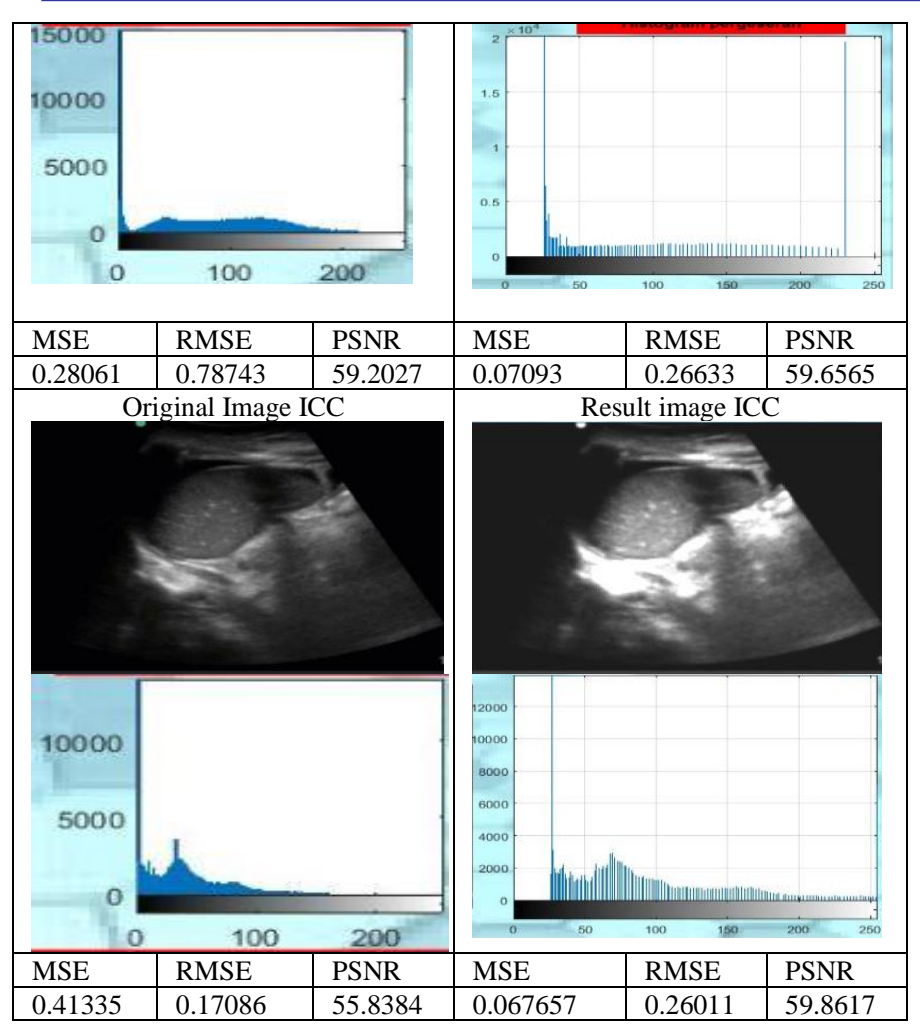

IV. CONCLUSION

Based on the results of the study, the following conclusions are obtained:

1. The color of the images can be changed to darker or brighter ones.

2. None of the values of MSE and RMSE exceeds 1, and the PSNR value is not less than $30 \mathrm{db}$.

3. The final processed image looks smoother than the initial preprocessed image.

\section{ACKNOWLEDGEMENT}

This research was funded by the Directorate of Research and Community Service at the Directorate General of Strengthening Research and Development of the Ministry of Research, Technology and Higher Education (ristekdikti) in accordance with the Research Contract of the 2018 Budget
Year and the Agreement / Contract Number: 010.20 / LP / UG / III / 2018

\section{REFERENCES}

[1] Lee, W.-L., 2013. “An Ensemble-Based Data Fusion Approach For Characterizing Ultrasonic Liver Tissue”. Appl. Soft Comput. 13(8) 3683-3692, 2013 J. Clerk Maxwell, A Treatise on Electricity and Magnetism, 3rd ed., vol. 2. Oxford: Clarendon, 1892, pp.68-73.

[2] Intensity Adjustment: Image Processing Toolbox. https://edoras.sdsu.edu/doc/matlab/toolbox/images/enhanc12.html

[3] I Gupta Deep, Anand R.S., Tyagi, Barjeev. 2012. "Enhancement Of Medical Ultrasound Images Using Non-Linear Filtering Based On Rational-Dilation Wavelet Transform”. WCECS. San Francisco, USA

[4] K. Wongsritong, K. Kittayaruasiriwat, F. Cheevasuvit, K. Dejhan, A Somboonkaew, "Contrast enhancement using multipeak histogram equalization with brightness preserving”, Circuit and System, 1998, IEEE APCCAS 1998. The 1998 IEEE Asia-Pacific Conference on 24 27 Nov.1998, pp. 455-458, 1998

[5] Tizhoosh, H.R., Michaelis, B. 1998 "Improvement of Image Quality Based on Subjective Evaluation and Fuzzy Aggregation techniques". EUFIT'98, vol. 2, 1998, pp. 1325-1329.

[6] Ogrenci, K. Bazargan NI. Sarrafzadeh, "Image analysis and partitioning for FPGA implementation of image restoration, Image Processing," IEEE Transaction, 2000, pp.346-355.

[7] Mittal, Neetu. 2012. "Automatic Contrast Enhancement of Low Contrast Images using MATLAB". International Journal of Advanced Research in Computer Science. Volume 3, No. 1, Jan-Feb 2012. ISSN No. 0976-5697. Available Online at www.ijarcs.info

[8] R Gonzales, Rafael C. Woods, Richard Eugene. 2008. "Digital Image Processing Using Matlab". Prentice Hall

[9] Blanchet, Gerrad, Charbit, Maurice. 2006. "Digital Signal And Image Processing Using Matlab”. (C) HERMES Science Europe Ltd, 2001 ( ISTE Ltd, 2006"M. Young, The Technical Writer's Handbook. Mill Valley, CA: University Science, 1989.

[10] Geertsma,Taco, Vallei, Ziekenhuis Gelderse, Hitachi Medical Systems Europe and the Radiology Department of the Gelderse Vallei Hospital in Ede in the Netherlands. 2017. "Ultrasound Cases: Info." http://www.ultrasoundcases.info/Cases-Home.aspx

[11] https://commons.wikimedia.org/wiki/Category:Ultrasound_images_of_ gallbladder

[12] https://commons.m.wikimedia.org/wiki/Category:Ultrasound_images_ of_livers

[13] Szeliski, Richard. 2010. "Computer Vision: Algorithms and Applications", September 3, 2010. 